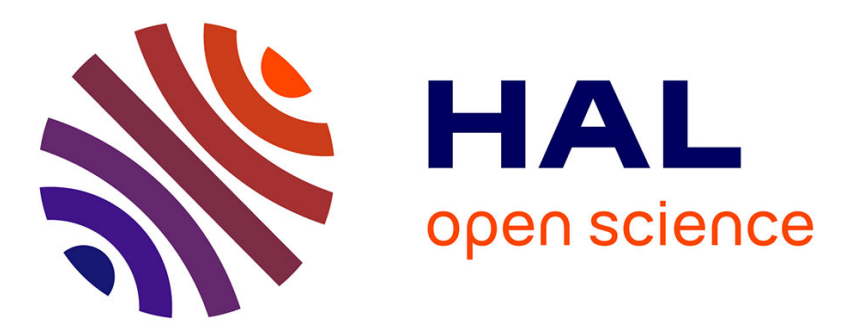

\title{
Chemically Triggered Ejection of Membrane Tubules Controlled by Intermonolayer Friction
}

\author{
J.-B. Fournier, Nada Khalifat, N. Puff, M. Angelova
}

\section{To cite this version:}

J.-B. Fournier, Nada Khalifat, N. Puff, M. Angelova. Chemically Triggered Ejection of Membrane Tubules Controlled by Intermonolayer Friction. Physical Review Letters, 2009, 102 (1), pp.018102. 10.1103/PhysRevLett.102.018102 . hal-02443688

\section{HAL Id: hal-02443688 \\ https://hal.sorbonne-universite.fr/hal-02443688}

Submitted on 6 Mar 2020

HAL is a multi-disciplinary open access archive for the deposit and dissemination of scientific research documents, whether they are published or not. The documents may come from teaching and research institutions in France or abroad, or from public or private research centers.
L'archive ouverte pluridisciplinaire HAL, est destinée au dépôt et à la diffusion de documents scientifiques de niveau recherche, publiés ou non, émanant des établissements d'enseignement et de recherche français ou étrangers, des laboratoires publics ou privés. 


\title{
Chemically Triggered Ejection of Membrane Tubules Controlled by Intermonolayer Friction
}

\author{
J.-B. Fournier, ${ }^{1}$ N. Khalifat, ${ }^{2}$ N. Puff, ${ }^{2}$ and M. I. Angelova ${ }^{2}$ \\ ${ }^{1}$ Laboratoire Matière et Systèmes Complexes (MSC), UMR 7057 CNRS and Université Paris Diderot-Paris 7 , \\ Bâtiment Condorcet, CC 7056, 75205 Paris, France \\ ${ }^{2}$ Université Pierre et Marie Curie-Paris 6, INSERM UMR S 893, CDR Saint-Antoine, F-75012 Paris, France
}

(Received 19 June 2008; published 7 January 2009)

\begin{abstract}
We report a chemically driven membrane shape instability that triggers the ejection of a tubule growing exponentially toward a chemical source. The instability is initiated by a dilation of the exposed monolayer, which is coupled to the membrane spontaneous curvature and slowed down by intermonolayer friction. Our experiments are performed by local delivery of a basic $p \mathrm{H}$ solution to a giant vesicle. Quantitative fits of the data give an intermonolayer friction coefficient $b \approx 2 \times 10^{9} \mathrm{~J} \mathrm{~s} / \mathrm{m}^{4}$. The exponential growth of the tubule may be explained by a Marangoni stress yielding a pulling force proportional to its length.
\end{abstract}

Lipid vesicles are model membranes made of amphiphilic molecules that self-assemble in water into closed bilayers. Although lacking membrane proteins and a cytoskeleton, they serve as simple models for cell membranes. Tubules are also ubiquitously present in living cells: They are constantly formed in the Golgi apparatus [1], in the endoplasmic reticulum [2], and in mitochondria [3]. Membrane tubules have also been shown to interconnect separate cells [4] and may provide a route for HIV transmission [5]. Tubules can be formed from model vesicles by applying external forces [6-8], using either hydrodynamical flows $[9,10]$, micropipettes $[11,12]$, optical tweezers [13], or molecular motors [14,15].

In this Letter, we report a curvature instability which is able to trigger the ejection of a tubule growing exponentially toward a chemical source. The mechanism is the following. A local lipid chemical modification in one monolayer modifies the preferred area per lipid, thereby inducing a local bilayer spontaneous curvature. Since only one monolayer is affected, the lateral redistribution of the lipids (monolayer expansion) is strongly slowed down by intermonolayer friction [16,17], and the instability develops into a large local deformation. When applied to a small enough surface, it triggers the ejection of a tubule aiming at the chemical source. We first discuss the dynamical shape instability theoretically, and then we describe an experimental realization of it. The question of the tubule dynamics is addressed ultimately.

Theoretical description of the instability.-Membranes consist of two monolayers ( \pm ), of free energy densities

$$
f^{ \pm}=\frac{1}{4} \kappa\left(c \pm c_{0}\right)^{2}+\frac{1}{2} k\left(\frac{n^{ \pm}}{n_{0}}-1 \pm e c\right)^{2},
$$

in the standard harmonic description of a symmetric membrane $[17,18]$. Here $c$ is the sum of the two principal curvatures of the bilayer midsurface $\mathcal{S}, n^{ \pm}$are the monolayer lipid densities relative to $\mathcal{S}$ (i.e., at the level of the extremities of the lipid tails), $\kappa$ and $c_{0}$ are the monolayer bending elastic constant and intrinsic spontaneous curva- ture, respectively, and $k$ is the stretching elastic constant. Note the density-curvature coupling: The actual monolayer spontaneous curvature $c_{0}^{+}$(which minimizes $f^{+}$) is affected by the density variations, i.e., $c_{0}^{+}=\left(\kappa / \kappa^{\prime}\right) c_{0}-$ $2 k e\left(n^{+} / n_{0}-1\right) / \kappa^{\prime}$, where $\kappa^{\prime}=\kappa+2 k e^{2}$. There lies the source of our instability.

To model the action of the chemical agent, we introduce two scalar fields $\epsilon^{ \pm}(\mathbf{r})$, representing the induced reduction of the optimal (equilibrium) lipid densities. As justified later, only $n_{0}$ is assumed to be chemically modified, not $c_{0}$. The stretching energy density becomes thus $\frac{1}{2} k\left[n^{ \pm} / n_{0}-\right.$ $\left.1+\epsilon^{ \pm}(\mathbf{r}) \pm e c\right]^{2}$. We assume further that only monolayer " $+"$ is affected, i.e., $\boldsymbol{\epsilon}^{-}(\mathbf{r})=0, \forall t$, and $\boldsymbol{\epsilon}^{+}(\mathbf{r}, t)=$ $\theta(t) \boldsymbol{\epsilon}^{+}(\mathbf{r})$, where $\theta(t)$ is the unit-step function. To account for the membrane tension $\sigma$, we consider that $n^{+}$and $n^{-}$ lie in the vicinity of $\bar{n} \neq n_{0}$, while the total number $N=$ $\bar{n} \mathcal{S}$ of lipids in each monolayer is fixed. In the small distortion limit, we describe the bilayer midsurface by a height function $z=h(\mathbf{r})$, where $\mathbf{r} \equiv(x, y)$, and we introduce the density fields $\rho^{ \pm}(\mathbf{r})$ defined as the relative excess-with respect to $\bar{n}$ - of the lipid densities projected onto the $(x, y)$ plane. Keeping only harmonic terms, with $d \mathcal{S} \simeq d x d y\left[1+\frac{1}{2}(\nabla h)^{2}\right], c \simeq \nabla^{2} h$, and $n^{ \pm} \simeq \bar{n}\left(1+\rho^{ \pm}\right) \times$ $\left[1-\frac{1}{2}(\nabla h)^{2}\right]$, we obtain the free energy

$$
\begin{aligned}
F \simeq & \int d x d y\left[\frac{\sigma}{2}(\nabla h)^{2}+\frac{\kappa}{2}\left(\nabla^{2} h\right)^{2}+\frac{k}{2}\left(\rho^{-}-e \nabla^{2} h\right)^{2}\right. \\
& \left.+\frac{k}{2}\left[\rho^{+}+e \nabla^{2} h+\theta(t) \epsilon^{+}(\mathbf{r})\right]^{2}\right],
\end{aligned}
$$

where $\sigma=k\left(1-\bar{n}^{2} / n_{0}^{2}\right), k$ and $e$ being slightly renormalized. Note that $\sigma$ coincides with $\partial F_{s} /\left.\partial \mathcal{S}\right|_{N}$, where $F_{s}=$ $\mathcal{S} k\left(\bar{n} / n_{0}-1\right)^{2}$ is the total stretching energy.

To determine the dynamical evolution of the membrane, we follow the analysis of Seifert and Langer [17]. For $t>$ 0 , the normal force density acting along the membrane is given by $p_{n}(\mathbf{r}, t)=-\delta F / \delta h(\mathbf{r})$, i.e.,

$$
p_{n}=-\kappa^{\prime} \nabla^{4} h+\sigma \nabla^{2} h-k e \nabla^{2}\left(\rho^{+}-\rho^{-}+\epsilon^{+}\right) .
$$


At $t=0$, since $h=\rho^{ \pm}=0$, there is a destabilizing force per unit area $\bar{p}_{n}=-k e \nabla^{2} \epsilon^{+}$. The two-dimensional (2D) pressure within each monolayer is given by $\pi^{ \pm}=(1+$ $\left.\rho^{ \pm}\right) \partial f^{ \pm} / \partial \rho^{ \pm}-f^{ \pm} \simeq k\left(\rho^{ \pm} \pm e \nabla^{2} h+\epsilon^{ \pm}\right)$, with $\epsilon^{-} \equiv 0$. We perform a linear stability analysis, assuming a modulated $\epsilon^{ \pm}(\mathbf{r})=E^{ \pm} e^{i q x}$, independent of time (for $t>0$ ), and setting $h(\mathbf{r}, t)=H(t) e^{i q x}$ and $\rho^{ \pm}(\mathbf{r}, t)=R^{ \pm}(t) e^{i q x}$. The dynamical equations consist of a Stokes-like tangential stress balance involving the lipid in-plane velocity fields $\mathbf{v}^{ \pm}(\mathbf{r}, t)=V^{ \pm}(t) e^{i q x} \mathbf{e}_{x}$, a normal stress balance, and a mass conservation equation [17]:

$0=-\eta_{2} q^{2} V^{ \pm}-k i q\left(R^{ \pm} \mp e q^{2} H+E^{ \pm}\right)-2 \eta q V^{ \pm} \mp b \Delta V$,

$0=-\left(\kappa^{\prime} q^{4}+\sigma q^{2}\right) H+k e q^{2}\left(R^{+}-R^{-}+E^{+}\right)-4 \eta q \partial_{t} H$,

$0=\partial_{t} R^{ \pm}+i q V^{ \pm}$

where $\Delta V=V^{+}-V^{-}$. The first equation displays the 2D viscous stress $\eta_{2} \nabla^{2} \mathbf{v}^{ \pm}$, the pressure gradient $-\nabla \pi^{ \pm}$, the viscous stress due to the $3 \mathrm{D}$ solvent motion, and the intermonolayer friction, respectively. The second one displays $p_{n}$ and the normal stress due to the $3 \mathrm{D}$ solvent motion, of normal velocity matching $\partial_{t} h$. Typical values of the parameters, used throughout, are $\kappa \simeq 10^{-19} \mathrm{~J}, k \simeq$ $0.1 \mathrm{~J} / \mathrm{m}^{2}, e \simeq 1 \mathrm{~nm}, \eta \simeq 10^{-3} \mathrm{~J} \mathrm{~s} / \mathrm{m}^{3}, \eta_{2} \simeq 10^{-9} \mathrm{~J} \mathrm{~s} / \mathrm{m}^{2}$ [17], and $b \simeq 10^{9} \mathrm{~J} \mathrm{~s} / \mathrm{m}^{4}[19,20]$.

Let us define $\bar{R}(t)=R^{+}+R^{-}$and $\hat{R}(t)=R^{+}-R^{-}$. To lighten the notations, we set $E^{+} \equiv \epsilon$, which is our central variable, the time-independent amplitude of the chemical modification. Eliminating $V^{ \pm}$in Eqs. (4) yields $\tau \partial_{t} \bar{R}=$ $-(\bar{R}+\epsilon)$, with $\tau=k^{-1}\left(\eta_{2}+2 \eta / q\right)$ and

$$
\partial_{t}\left(\begin{array}{c}
H \\
\hat{R}
\end{array}\right)=-\left(\begin{array}{cc}
\frac{\kappa^{\prime} q^{3}+\sigma q}{4 \eta} & -\frac{k e q}{4 \eta} \\
-\frac{k e q^{4}}{b} & \frac{k q^{2}}{2 b}
\end{array}\right)\left(\begin{array}{c}
H \\
\hat{R}+\epsilon
\end{array}\right),
$$

where we have assumed $\eta_{2} q^{2} \ll b$ and $\eta q \ll b$. The initial conditions are $\bar{R}(0)=\hat{R}(0)=H(0)=0$. We see that $\bar{R}(t)$ relaxes to $\bar{R}(\infty)=-\epsilon$ quasi-instantaneously; indeed, given the above parameters, $\tau \lesssim 0.5 \mu$ s even at a large wavelength such that $\pi / q \simeq 100 \mu \mathrm{m}$. Since the coupled dynamics of $\hat{R}$ and $H$ will turn out to be much slower, we may consider that $\bar{R}\left(0^{+}\right)=-\epsilon$, yielding $R^{ \pm}\left(0^{+}\right)=-\epsilon / 2$ [Figs. 1(a)-1(c)].

The subsequent evolution [Figs. 1(c)-1(e)] is controlled by Eq. (5). Its eigenvalues yield two relaxations rates: a slow one $\gamma_{1}$ and a faster one $\gamma_{2}>\gamma_{1}$. The solution gives

$$
H(t)=\epsilon \frac{k e q}{4 \eta} \frac{e^{-\gamma_{1} t}-e^{-\gamma_{2} t}}{\gamma_{2}-\gamma_{1}},
$$

attaining $\quad H_{\max }=k e q \epsilon / 4 \eta \gamma_{2} \times\left(\gamma_{1} / \gamma_{2}\right)^{\gamma_{1} /\left(\gamma_{2}-\gamma_{1}\right)}$ at $t_{\max }=\ln \left(\gamma_{2} / \gamma_{1}\right) /\left(\gamma_{2}-\gamma_{1}\right)$ and converging to $H(\infty)=0$ and $\hat{R}(\infty)=-\epsilon$ (see Fig. 2). Note that $t_{\max }$ may be comparable with or much smaller than the total decay time $\gamma_{1}^{-1}$. The values of $\gamma_{1}$ and $\gamma_{2}$ were discussed for $\sigma=0$ in Ref. [17]. For $\sigma \gtrsim \sigma_{c}=4 k^{2} \eta^{2} / b^{2} \kappa^{\prime}\left(\approx 10^{-8} \mathrm{~J} / \mathrm{m}^{2}\right.$ with the above values), there are two regimes: $\left(\gamma_{1}, \gamma_{2}\right) \simeq$ $\left(k q^{2} / 2 b, q \sigma / 4 \eta\right)$ for $q \ll q_{3}=\sqrt{\sigma / \kappa^{\prime}}$ and $\left(\gamma_{1}, \gamma_{2}\right) \simeq$ a)

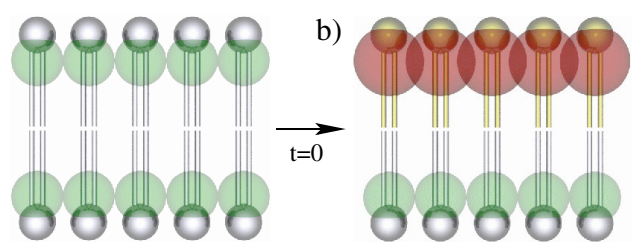

c)

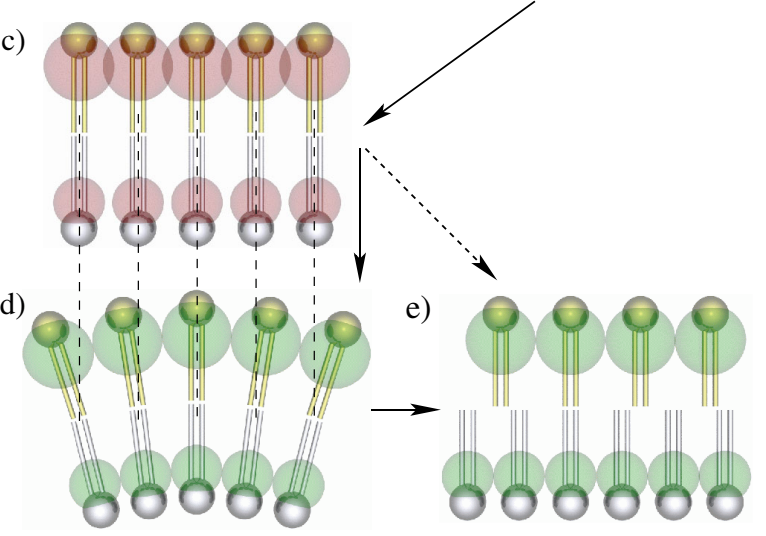

FIG. 1 (color online). (a) Initial bilayer. (b) At $t=0$, a lipid chemical modification (gold) induces an effective compression indicated by the red spheres on the "neutral surface." (c) In a very short time $\tau \lesssim 1 \mu \mathrm{s}$, the lipids slide in both monolayers: Half of the compression of the upper lipids is relaxed, while the slippage-free drag induces some dilation in the lower monolayer. (d) The overall stretching energy may be relaxed at fixed density by curving the bilayer. At the macroscopic scale, this curvature instability is quicker than the intermonolayer slippage necessary to reach the final equilibrium state (e).

$\left(k q^{2} \kappa / 2 b \kappa^{\prime}, \kappa^{\prime} q^{3} / 4 \eta\right)$ for $q \gg q_{3}$. Assuming $\gamma_{2} \gg \gamma_{1}$, we may approximate (see Fig. 2):

$$
\begin{gathered}
H_{\max } \approx \frac{k e \epsilon}{\kappa^{\prime} q^{2}+\sigma}\left(\frac{2 k q \eta / b}{\kappa^{\prime} q^{2}+\sigma}\right)^{(2 k q \eta / b) /\left(\kappa^{\prime} q^{2}+\sigma\right)}, \\
t_{\max } \approx \frac{2 b}{k q^{2}} \frac{\ln \left(\frac{\kappa q^{2}+\sigma}{2 k q \eta / b}\right)}{\frac{\kappa q^{2}+\sigma}{2 k q \eta / b}-1} .
\end{gathered}
$$

The prefactor in $H_{\max }$ corresponds to the equilibrium amplitude that would be reached if the densities were frozen. As can be seen in Fig. 2, $H_{\max }$ and $t_{\max }$ may easily reach macroscopic values for perturbations on a scale larger than $10 \mu \mathrm{m}$ when $\sigma \lesssim 10^{-6} \mathrm{~J} / \mathrm{m}^{2}$.

Experiments. - Giant unilamellar vesicles in the fluid phase were formed at $25^{\circ} \mathrm{C}$ in a buffer at $p \mathrm{H} 7.4$ by electroformation [21] from a mixture of egg yolk phosphatidylcholine (EYPC) and brain phosphatidylserine (PS) with EYPC/PS 90:10 mol/mol. The chemical modification of the membrane was achieved by locally delivering to the membrane outer leaflet a basic solution of $\mathrm{NaOH}(1 M, p \mathrm{H}$ 13) with a micropipette of diameter $0.3 \mu \mathrm{m}$ controlled by an Eppendorf Femtojet pressure device and Narishige micromanipulator. Estimations based on visualizing the flux from the micropipette and taking into account the dilution of $\mathrm{NaOH}$ in the giant unilamellar vesicle's formation buffer 

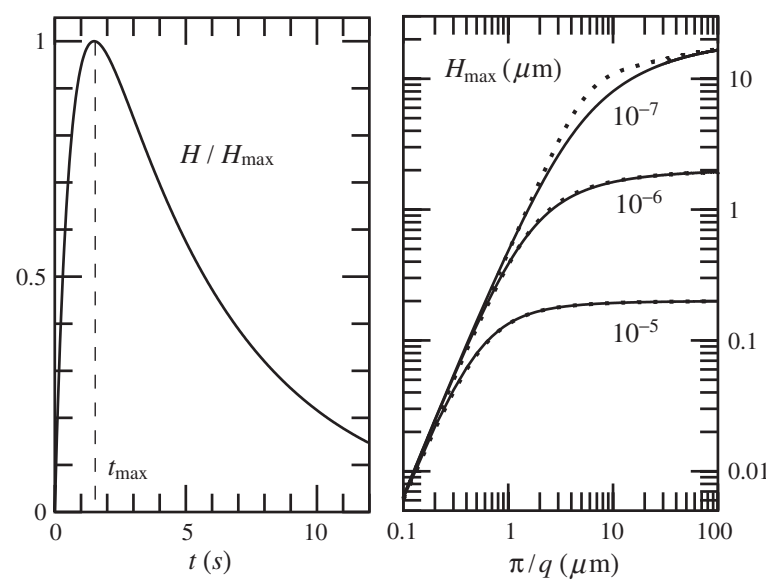

FIG. 2. (Left) Time evolution of the normalized amplitude of the membrane undulation. Here $\epsilon=0.02, \pi / q=50 \mu \mathrm{m}, \sigma=$ $10^{-7} \mathrm{~J} / \mathrm{m}^{2}$, and all of the other parameters have the values given in the text, yielding $H_{\max } \simeq 14.7 \mu \mathrm{m}$. (Right) $H_{\max }$ as a function of the width of the imposed perturbation. The plain lines are exact values, the dotted lines correspond to Eq. (7), and the numbers give the membrane tension in $\mathrm{J} / \mathrm{m}^{2}$.

$(p \mathrm{H}$ 7.4) yield, at the deformation onset, a $p \mathrm{H}$ ranging from 8.2 to 9.2 on the vesicle's membrane. The vesiclepipette system was observed under a Zeiss Axiovert 200M inverted microscope equipped with a CCD camera (Cool SNAP HQ) and Metamorph system.

In a first set of experiments (Fig. 3), we slowly approach the pipette while it is expelling a few picoliters per second of the basic solution under an injection pressure of $100 \mathrm{hPa}$. At a distance of $\simeq 25 \mu \mathrm{m}$, a two-step process

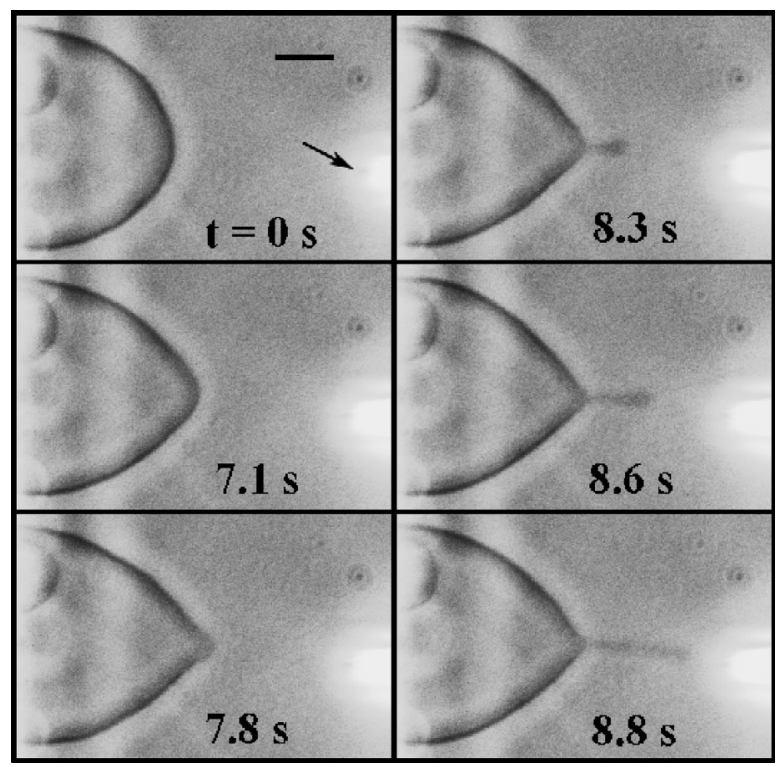

FIG. 3. Increasing deformation of a giant unilamellar vesicle (frames 7.1-7.8 s) and spontaneous ejection of a tubule (frames 8.3-8.8 s) induced by the local delivery of a basic $\mathrm{NaOH}$ solution (1M, $p \mathrm{H} \mathrm{13)}$ by a micropipette (arrow). The final tube velocity is $\simeq 40 \mu \mathrm{m} \mathrm{s}^{-1}$. Scaled bar $10 \mu \mathrm{m}$. occurs: (i) A smooth deformation of the vesicle starts to develop toward the pipette (i.e., opposite to the flow). At this point we stop the pipette while it keeps expelling the solution, and we observe the increasing deformation. After a critical amplitude, (ii) a tubule is suddenly expelled, which aims at the pipette. The time evolution $L(t)$ of the tubule length is exponential, as shown in the inset in Fig. 4. In a second set of experiments, we try to perform a timestep chemical modification (Fig. 4). We quickly approach the pipette without any injection, then we inject the solution for a time $\Delta t \simeq 3 \mathrm{~s}$, and we quickly withdraw the pipette before the ejection of any tubule. On the other hand, we checked that no deformation occurs if only buffer solution is injected. The time evolution $H(t)$ of the deformation amplitude is shown in Fig. 4.

Comparison between experiment and theory.-The phospholipids EYPC and PS have $p \mathrm{H}$-sensitive ionizable head groups. In particular, PS should become more negatively charged upon significantly increasing the $p \mathrm{H}$ above 7.4. Hence, the delivered solution should effectively increase the repulsion between the lipids of the exposed monolayer, which we model by a parameter $\epsilon>0$. For a variation of the effective head group area of a few percent, i.e., $\epsilon \approx 10^{-2}$, it is reasonable to neglect the variation of the intrinsic spontaneous curvature $c_{0}$. Indeed, assuming $c_{0}^{-1} \approx 50 \mathrm{~nm}$, one may check from Eq. (1) that the variation $\delta c_{0}$ that would match the effect of $\epsilon$ is such that $\delta c_{0} / c_{0} \simeq 2 k e \epsilon /\left(\kappa c_{0}\right) \simeq 100 \epsilon \approx 1$, i.e., is unrealistic. We also exclude possible osmotic effects, the latter producing membrane deformations in the direction opposite to the concentration gradient [22] and flip-flop effects, their characteristic times being of several hours.

Let us first discuss the instability, step (i). Our model assumes a spatially modulated chemical modification, while in our experiment we deliver the basic solution

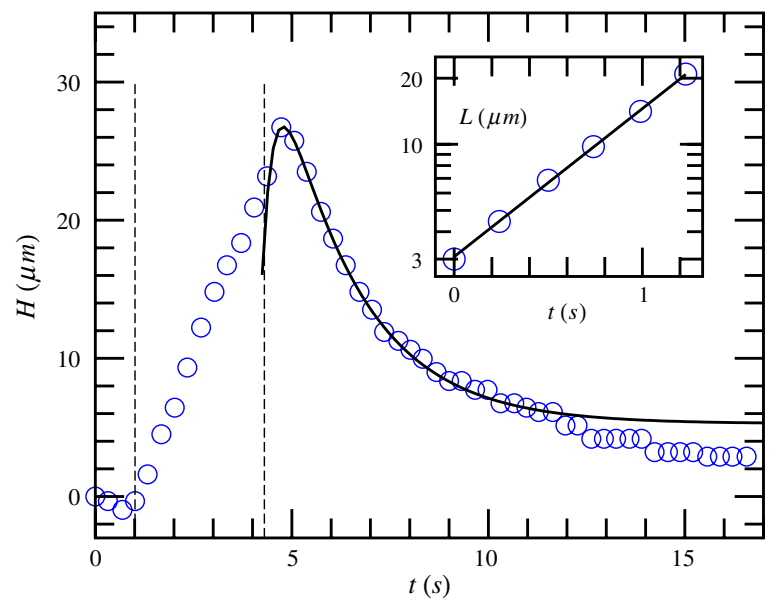

FIG. 4 (color online). Time evolution of the vesicle deformation, measured in front of the pipette, for an injection limited to the time interval between the vertical dashed lines. The fit corresponds to Eq. (6). (Inset) Exponential time evolution $L(t)$ of the tubule length in Fig. 3. 
locally over a membrane region of size $D \simeq 20 \mu \mathrm{m}$. To make the correspondence, we take $q \simeq \pi / D$, and we identify $\epsilon$ with the maximum value $\epsilon^{+}\left(\mathbf{r}_{0}\right)$ produced in front of the pipette. In the experiment of Fig. 4, if $t_{\max } \ll \Delta t$, the linear increase of $H(t)$ during the injection time $\Delta t$ (Fig. 4) may be interpreted as a linear increase of the resulting $\epsilon^{+}\left(\mathbf{r}_{0}, t\right)$. It is then reasonable to fit the relaxation of $H(t)$ in Fig. 4 with Eq. (6). The quality of this fit supports our model. The faster relaxation at large times may be accounted for by the readsorption of protons and the neutralization of the excess membrane negative charge as the basic solution diffuses away in the bulk. The fit yields $\gamma_{1} \approx 0.5 \mathrm{~s}^{-1}$ and $\gamma_{2} \approx 3 \mathrm{~s}^{-1}$ (justifying $t_{\max } \ll \Delta t$ ). Anticipating $\sigma \gtrsim \sigma_{c}$ and $q \ll q_{3}$, we use the relations $\gamma_{1} \approx k q^{2} / 2 b$ and $\gamma_{2} \approx q \sigma / 4 \eta$ to determine $\sigma$ and $b$, assuming $q \simeq 1.6 \times 10^{5} \mathrm{~m}^{-1}, \eta \approx 10^{-3} \mathrm{~J} \mathrm{~s} / \mathrm{m}^{3}$, and $k \approx$ $0.1 \mathrm{~J} / \mathrm{m}^{2}$. This gives $\sigma \approx 10^{-7} \mathrm{~J} / \mathrm{m}^{2}$ (reasonable given the floppy nature of the vesicle) and $b \approx 2 \times 10^{9} \mathrm{~J} \mathrm{~s} / \mathrm{m}^{4}$, compatible with Ref. [19]. Finally, we may deduce the experimental value of $\epsilon$ from the value $H_{\max } \simeq 30 \mu \mathrm{m}$. Using the theoretical expression for $H_{\max }$ given after Eq. (6) and assuming $e \approx 10^{-9} \mathrm{~m}$, we obtain $\epsilon \approx$ $3 \times 10^{-2}$. We conclude that our model describes step (i) quantitatively.

Let us now discuss the nucleation and growth of the tubule, step (ii). Our instability produces a destabilizing pressure $\bar{p}_{n}=-k e \nabla^{2} \epsilon^{+}$on the membrane. Can the corresponding force $f_{n} \approx \bar{p}_{n} D^{2}$ overcome the force $f_{t}=$ $2 \pi \sqrt{2 \kappa \sigma}[6,7]$ required to pull a tubule? From $\bar{p}_{n} \approx$ $k e(\pi / D)^{2} \epsilon$, we find that this requires $\epsilon>\epsilon_{c}$, with $\epsilon_{c}=$ $\sqrt{8 \kappa \sigma} / \pi k e \simeq 3 \times 10^{-3}$ for $\sigma \simeq 10^{-6} \mathrm{~J} / \mathrm{m}^{2}$ (floppy vesicle). This threshold is indeed lower than the experimental $\epsilon$ deduced from our fit. Pulling a tubule, however, requires overcoming a force barrier increasing linearly with $D$ [8]. Accordingly, we find that the ejection of the tubule occurs only when the membrane gets close enough to the pipette (focalized flow). Considering that the tubule growth involves the presence of an interface submitted to a physicochemical gradient $(p \mathrm{H})$, it is natural to consider the possibility of surface tension gradients, i.e., of a Marangoni effect. Indeed, the chemical gradient implies a $\nabla \epsilon^{+}$in the outer monolayer, which produces the Marangoni stress $\boldsymbol{\nabla} \pi^{+}=k \boldsymbol{\nabla} \epsilon^{+}$. The resulting pulling force is $F=2 \pi r \times$ $k\left|\nabla \epsilon^{+}\right| L$, where $r$ is the tube radius. Note that $F$ increases linearly with $L$, which may explain the exponential growth. Indeed, for short enough tubes, the main dissipation occurs at their base, yielding a restoring friction force $F_{v}=$ $\lambda d L / d t$ independent of $L$ [16]. Equating $F$ and $F_{v}$ yields $L(t) \propto \exp (\gamma t)$, with $\gamma=2 \pi r k|\nabla \epsilon| / \lambda$. Experimentally, we find $\gamma \simeq 1.5 \mathrm{~s}^{-1}$ (Fig. 4). A model for $\lambda$ (hence a measurement of $|\nabla \epsilon|$ ) in such fast pulling conditions involving nonrelaxed lipid densities is, however, outside the scope of this Letter.

Finally, note that our situation clearly differs from that of Ref. [23], where tubulation is believed to result mainly from a modification of the intrinsic spontaneous curvature $\left(c_{0}\right)$ of the outer monolayer, as induced by the insertion of hydrophobic polymer sidegroups: Our tubes show no pearling, and their growth is 2 orders of magnitude faster and exponential rather than linear. Besides, our tubes instantly follow the imposed gradient's direction, while in Ref. [23] it was possible to increase the tube length by injecting polymers at its base.

In conclusion, we have shown that a modification of the preferred area per lipid occurring in one monolayer may yield-even if it affects a small area-a large local transient deformation, because the lateral distribution of the resulting effective spontaneous curvature (of the areadifference type) is slowed down by intermonolayer friction. This phenomenon might occur in biological processes. The potential nonlinear development of this instability, i.e., the spontaneous, exponential growth of a tubule toward a chemical source, can be viewed as a new kind of chemotaxis. In particular, membrane deformations guided by a local $p \mathrm{H}$ gradient might be involved in cell signaling processes. Our experiment may also be viewed as a new method to measure the intermonolayer friction coefficient.

We thank C. Sykes for valuable discussions. This work was supported by the "Dynamics of complex systems" Federation (FED21/UPMC).

[1] N. Sciaky et al., J. Cell Biol. 139, 1137 (1997).

[2] C. Lee and L. B. Chen, Cell 54, 37 (1988).

[3] C. A. Mannella et al., Intl. Union Biochem. Mol. Biol. Life 52, 93 (2001).

[4] A. Rustom et al., Science 303, 1007 (2004).

[5] S. Sowinski et al., Nat. Cell Biol. 10, 211 (2008).

[6] I. Derényi, F. Jülicher, and J. Prost, Phys. Rev. Lett. 88, 238101 (2002).

[7] T. R. Powers, G. Huber, and R. E. Goldstein, Phys. Rev. E 65, 041901 (2002).

[8] G. Koster, A. Cacciuto, I. Derényi, D. Frenkel, and M. Dogterom, Phys. Rev. Lett. 94, 068101 (2005).

[9] R. E. Waugh, Biophys. J. 38, 29 (1982).

[10] O. Rossier et al., Langmuir 19, 575 (2003).

[11] E. Evans, H. Bowman, A. Leung, D. Needham, and D. Tirrel, Science 273, 933 (1996).

[12] N. Khalifat et al., Biophys. J. 95, 4924 (2008).

[13] D. Raucher and M. P. Sheetz, Biophys. J. 77, 1992 (1999).

[14] G. Koster, M. VanDuijn, B. Hofs, and M. Dogterom, Proc. Natl. Acad. Sci. U.S.A. 100, 15583 (2003).

[15] C. Leduc et al., Proc. Natl. Acad. Sci. U.S.A. 101, 17096 (2004).

[16] E. Evans and A. Yeung, Chem. Phys. Lipids 73, 39 (1994).

[17] U. Seifert and S. A. Langer, Europhys. Lett. 23, 71 (1993).

[18] W. Helfrich, Z. Naturforsch. C 28, 693 (1973).

[19] T. Pott and P. Méléard, Europhys. Lett. 59, 87 (2002).

[20] S. A. Shkulipa, W. K. den Otter, and W. J. Briels, Phys. Rev. Lett. 96, 178302 (2006).

[21] M.I. Angelova and D.S. Dimitrov, Faraday Discuss. Chem. Soc. 81, 303 (1986).

[22] J. Nardi, R. Bruinsma, and E. Sackmann, Phys. Rev. Lett. 82, 5168 (1999).

[23] I. Tsafir et al., Phys. Rev. Lett. 91, 138102 (2003). 